\title{
Critical behaviour of thin films with quenched impurities
}

\author{
L. Craco $^{\dagger}$, L. De Cesare ${ }^{\ddagger}$, I. Rabuffo ${ }^{\ddagger}$, I. P. Takov*, and D. I. Uzunov ${ }^{*, \dagger, \ddagger, \uparrow}$ \\ $\dagger$ Max-Planck-Institut für Physik Komplexer Systeme, Aussenstelle Stuttgart, \\ Heisenbergstrasse 1, D-70569 Stuttgart, Federal Republic of Germany. \\ $\ddagger$ Dipartimento di Scienze Fisiche "E. R. Caianiello", Università di Salerno, \\ I-84081 Baronissi, Salerno, Italy, and Istituto Nazionalle per la Fisica della Materia, \\ Unità di Salerno, Italy. \\ * CPCM Laboratory, G. Nadjakov Institute of Solid State Physics, \\ Bulgarian Academy of Sciences, BG-1784 Sofia, Bulgaria. \\ ฯ Corresponding author.
}

Key words: finite size scaling, phase transition, quanched disorder, thin films, renormalization group, dimensional crossover, critical phenomena.

PACS: 05.70.Jk, 64.60.Ak, 64.60.Fr, 68.35.Rh

\begin{abstract}
The critical behaviour of thin films containing quenched random impurities and inhomogeneities is investigated by the renormalization-group method to the one-loop order within the framework of the $n$-component $\phi^{4}$-model. The finite-size crossover in impure films has been consdered on the basis of the fundamental relationship between the effective dimensionality $D_{\text {eff }}$ and the characteristic lengths of the system. The fixed points, their stability properties and the critical exponents are obtained and analyzed, using a $\tilde{\epsilon}=\left(4-D_{\text {eff }}\right)$-expansion near the effective spatial dimensionality $D_{\text {eff }}$ of the fluctuation modes in $D$-dimensional hyperslabs with two types of quenched impurities: point-like impurities with short-range random correlations and extended (linear) impurities with infinite-range random correlations long the spatial direction of the small size. The difference between the critical properties of infinite systems and films is demonstrated and investigated. A new critical exponent, describing the scaling properties of the thickness of films with extended impurities has been deduced and calculated. A special attention is paid to the critical behaviour of real impure films $(D=3)$.
\end{abstract}




\section{Introduction}

The development of the theory of phase transitions in thin films in the last decades has been based mainly on statistical methods 11 such as, for example, the lattice mean-field (MF) approximation [2, 3], the phenomenological Landau-Ginzburg (LG) approach [4, the general scaling theory [ [, 6], and renormalization-group (RG) schemes of calculation [7, 8]. The general approach to the description of finite-size (FS) systems having different geometries, including the film geometry, is the Fisher FS scaling theory [5, 6]. Within this framework the field-theoretical RG methods are applied to the investigation of the possible types of critical behaviour and the calculation of important characteristics like the critical exponents, scaling amplitudes and crossover functions [7, 8]. The calculations are performed for systems with a general spatial dimensionality $D$. The predictions for real systems $(D=3)$ are made by extrapolations from the corresponding $\epsilon=\left(D_{U}-D\right)$-expansions, where $D_{U}$ is the upper critical dimensionality [9]). The method of $\epsilon$-expansions is widely and successfully applied to the investigation of the critical properties of FS and infinite systems. It is proven to be particularly convenient for the study of complex models of real substances, where the competing effects play a substantial role.

For FS systems one of the most interesting problems is the dimensional FS crossover (FSC) from the usual $D$-dimensional critical behaviour to the corresponding $d=\left(D-D_{0}\right)$ dimensional critical behaviour when the finite sizes $L_{0 i}\left(i=1, \ldots, D_{0}\right)$ are less than the correlation length $\xi \sim\left(T-T_{c}\right)^{-\nu} ; T_{c}$ is the critical temperature [5, 7]. As $\xi \rightarrow \infty$ for $T \rightarrow T_{c}$, the FSC $(D \rightarrow d)$ in the asymptotic critical behaviour occurs always provided the number $d$ of "infinite" dimensions $L_{i} \gg \xi,(i=1, \ldots, d)$ is larger than the lower borderline (critical) dimensionality $D_{L}[9]$.

In this paper we shall study the FSC in $D$-dimensional hyperfilms $\left(D_{0}=1, d=D-1\right)$ of thickness $L_{0}$ with quenched random impurities and inhomogeneities [9, 10, which can be described by a properly chosen $\phi^{4}$-model of critical phenomena with an $n$-component fluctuation field (order parameter) $\phi(x)=\left\{\phi_{\alpha}(\vec{x}), \alpha=1, \ldots, n\right\}$. A special attention will be paid to the case of real films $(D=3)$. Thus we shall investigate a complex system with three competing effects: the thermal fluctuations, the FS, and the disorder. The main features of the critical behaviour in such complex systems can be described by the Wilson-Fisher RG recursion relations in the one-loop approximation [9]. The one-loop approximation leads to a reliable prediction of the possible types of critical behaviour, namely, of the fixed points (FP) of the RG equations, their stability properties, and of the first corrections to the MF values of the critical exponents. We shall perform this programme for basic models of random impurities by introducing a new approach to the RG investigation of the FS effects.

The RG investigations of films reveal the two limiting cases of the FSC: (i) thick (quasi$D$-dimensional) films, where the ratio $y=\left(L_{0} / \xi\right)$ tends to infinity $(y \gg 1)$, and (ii) thin (quasi- $d$-dimensional) films, where the ratio $y$ tends to zero $(y \ll 1)$. In the former 
case the upper borderline dimensionality is $D_{U}=4$, whereas in the latter case this dimensionality is $D_{U}=5$ [7, 8]. The $\epsilon$-expansions for the cases (i) and (ii) are performed for $\epsilon=(5-D)=(4-d)$ and $\epsilon=(4-D)=(3-d)$, respectively. Here we shall present an unified RG investigation based on an $\tilde{\epsilon}=\left(4-D_{\text {eff }}\right)$-expansion, where $D_{\text {eff }} \in[d, D]$ is the effective dimensionality of the fluctuation modes $\phi(\vec{x})$ [11]. For this aim we shall apply the so-called integration over dimensionalities $\delta\left(=D_{\text {eff }}-d\right)$ less than unity. This approach has been recently used to perform a $\delta \leq 1$-integration over the time axis for problems of the quantum critical behaviour in disordered superfluids [12. Working in a similar way one may obtain a description of the critical behaviour in impure thin films for any effective spatial dimensionality $D_{\text {eff }} \in[d, D]$.

The term effective spatial dimensionality $\left(D_{\text {eff }}\right)$ has been recently introduced [11] in an attempt for a description of the FSC as a smooth variation of the effective dimensionality of the fluctuation modes $\phi(\vec{x})$ with the variations of the ratio $y=\left(L_{0} / \xi\right)$. Although the precise dependence $D_{\text {eff }}(y)$ cannot be easily obtained, it seems intuitively obvious that in thin films $D_{\text {eff }}(y) \sim d$ for $y \ll 1$ (the case of thin films) and $D_{\text {eff }}(y) \sim D=(d+1)$ for $y \gg 1$ (the case of thick films or, equivalently, of almost infinite systems).

The random impurities and inhomogeneities produce the effect of "random critical temperature" $T_{c}(\vec{x})$ which depends on the spatial vector $\vec{x}$ [9, 10, 13, 14]. This type of (nonequilibrium, quenched) disorder is investigated by choosing a convenient, usually, Gaussian distribution for the random (nonequilibrium) temperature $T_{c}(\vec{x})$. The critical behaviour of infinite systems both for point [13] and extended [14] impurities is well known; see also [9, 10]. We consider the FS effect on the critical behaviour in films with these basic types of disorder.

Within the model of point impurities with spatially isotropic short-range random correlations the FSC $(D \rightarrow d)$ can be easily proven. Besides, we demonstrate that the critical exponents are smooth functions of $D_{\text {eff }}$ and, hence, of the ratio $y=\left(L_{0} / \xi\right)$. We shall show that the impure critical behaviour predicted by predicted by T. C. Lubensky [13] for infinite systems with point impurities occurs in thin films for any effective dimensionality $D_{\text {eff }}>2$, i.e. for any ratio $y=\left(L_{0} / \xi\right)$.

The disorder of type extended impurities is described by infinitely-ranged random correlations along one or more spatial directions. For systems of a film geometry the appropriate choice of extended impurities is the case of one-dimensional (line) impurities orientated along the direction of the small size $L_{0}$ and randomly distributed along the other spatial directions. This disorder is described $b$ a modification of the model of short-range correlated point impurities in which the short-range correlations along the small size are substituted with infinite-range correlations. The length scale of the latters is much larger than both the correlation length $\xi$ and the thickness $L_{0}$. So the strongly correlated along the small size point impurities behave like continuous uniform strings. In regards to the critical behaviour this disorder acts like point impurities with a short-range random distribution along the large (infinite) dimensions $L_{i}$ and an uniform distribution along the 
small size $L_{0}$. The anisortopy of the random correlations lead to a quite unusual critical behaviour of the impure films. This behaviour is described in details. As a result of our analysis, we have identifies a new critical exponent describing the scaling law of the thickness $L_{0}$ of the film. This exponent is analogous to the dynamical critical exponent $z$ in disordered classical [15] and quantum systems [16].

In Section 2 we present the model of consideration. The specific FS features of the model are discussed in Section 3. The integration in dimensionalities less than unity and the effective dimensionality $D_{\text {eff }}$ are introduced in Section 4 . The RG investigations presented in Sections 5 and 6 rely upon the basic problems discussed in Section 3 and 4. In Section 7 we summarize the results and discuss their applicability to real threedimensional $(3 D)$ films. The analogy between the FSC in thin films and the classical-toquantum crossover [17] at low temperatures is also disenssed.

\section{$2 \quad$ Model}

We shall use the usual $\phi^{4}$-Hamiltonian $\left(\mathcal{H}=H / T, k_{B}=1\right)$ of the $n$-component fluctuation field $\phi(\vec{x})$. We shall stick to the usual notations [9, 10, 13] in which the Hamiltonian $\mathcal{H}$ can be written in the form

$$
\mathcal{H}=\frac{1}{2} \int d^{D} x\left\{(\nabla \phi)^{2}+r(\vec{x}) \phi^{2}+2 u \phi^{4}\right\}
$$

In Eq. (11), $u>0, r(\vec{x})=r+\varphi(\vec{x})$, where $\varphi(\vec{x})$ is a random function intended to describe the disorder effects and $r=\alpha_{0}\left(T-T_{c 0}\right) / T_{c 0}$ is the usual Landau parameter represented by the (bare) critical temperature $T_{c 0}$ of the pure systems $[\varphi(\vec{x}) \equiv 0]$. The random function $\varphi(\vec{x})$ obeys the Gaussian distribution [9, 10]:

$$
\left[\varphi(\vec{x}) \varphi\left(\vec{x}^{\prime}\right)\right]_{R}=\Delta \delta\left(\vec{x}-\vec{x}^{\prime}\right)
$$

$(\Delta \geq 0)$. The function $\varphi(\vec{x})$ is related to the "random" critical temperature $T_{c 0}(\vec{x})$ in the following way: $T_{c 0}(\vec{x})=T_{c 0}\left[1-\varphi(\vec{x}) / \alpha_{0}\right]$. The true (renormalized) critical temperature $T_{c}$ will be a function of $T_{c 0}$ and the renormalized values of the interaction parameters $u$ and $\Delta$. The averages $\langle A\rangle_{R}$ of the physical quantities $A[\varphi(\vec{x})]$ are defined by the functional integral

$$
\langle A\rangle_{R}=\int \prod_{\vec{x}} \mathcal{D} \varphi(\vec{x}) e^{-\frac{1}{2 \Delta} \int d^{D} x \varphi^{2}(\vec{x})} A[\varphi(\vec{x})] .
$$

We shall work with periodic boundary conditions. This means that we neglect the surface energy which is important in the investigation of other properties of thin films. This way of treatment gives the opportunity to investigate the net effect of the FS $L_{0}$ on the critical behaviour of the impure films.

The $\mathrm{RG}$ investigation is performed in the space of the wave vectors. Taking into account the lattice structure, the components $q_{\nu}$ of the $D$-dimensional wave vector $\vec{q}=\left(q_{\nu} ; \nu=\right.$ 
$0,1, \ldots, d)$ are confined in the first Brillouin zone $\left(-\pi / a<q_{\nu} \leq \pi / a\right)$. However, because of the long-wavelength approximation (LWLA), $a q_{\nu} \ll \pi$, which is unavoidably included in field models like the Hamiltonian (11), the upper cutoff $\Lambda$ of the wave vector $\vec{q}$ is much smaller than $(\pi / a)$, namelly, $\Lambda=\gamma(\pi / a)$, where $\gamma \ll 1$. While the LWLA approximation does not introduce restrictions on the dimensions $L_{i}$ which are initially supposed to be almost infinite $\left(L_{i} \gg \xi\right.$ for any $\left.a<\xi<\infty\right)$, the same approximation restricts the variations of the thickness: $a \ll L_{0}$. Therefore our consideration is confined within the latter condition and our results could not be extended to exactly two-dimensional $(2 D)$ films (mono-atomic layers). It is convenient to write the $D$-dimensional wave vector $\vec{q}$

in the form $\vec{q}=\left(k_{0}, \vec{k}\right)$, where the wave number $k_{0}=\left(2 \pi n_{0} / L_{0}\right)$ with $n_{0}=0, \pm 1, \ldots$, corresponds to the thickness $L_{0}$, and the $d$-dimensional wave vector $\vec{k}$ with components $k_{i}=\left(2 \pi n_{i} / L_{i}\right),\left(n_{i}=0, \pm 1, \ldots,\right)$ corresponds to the $d$-dimensional "subsystem" of the film.

The short-range random correlations (2) correspond to point-like random impurities and inhomogeneities which are equally distrubuted along all $D$ directions. In the space of the wave vectors $\vec{q}$ the Eq. (2) takes the form

$$
\left[\varphi(\vec{q}) \varphi\left(\vec{q}^{\prime}\right]_{R}=\Delta \delta\left(\vec{q}, \vec{q}^{\prime}\right)\right.
$$

where $\delta(x, y)$ is the Kronecker symbol. The disorder of type "extended impurities" is described by the Gaussian distribution given by

$$
\left[\varphi(\vec{r}) \varphi\left(\vec{r}^{\prime}\right)\right]_{R}=\Delta \delta\left(\vec{r}-\vec{r}^{\prime}\right)
$$

where the vector $\vec{r}$ lays in the $d$-dimensional subsystem; as given by the definition $\vec{x}$ by $\vec{x}=\left(x_{0}, \vec{r}\right)$. In the $q$-space, the Eq. (5) takes the form

$$
\left[\varphi(\vec{k}) \varphi\left(\vec{k}^{\prime}\right)\right]_{R}=\Delta \delta\left(\vec{k}, \vec{k}^{\prime}\right)
$$

or, equivalently,

$$
\left[\varphi(\vec{q}) \varphi\left(\vec{q}^{\prime}\right)\right]_{R}=\Delta \delta\left(\vec{k}, \vec{k}^{\prime}\right) \delta\left(0, \vec{k}_{0}\right) \delta\left(0, \vec{k}_{0}^{\prime}\right) .
$$

These models of impure systems have been briefly explained in Section 1. Besides, they are described well in several preceding papers [10, 13] and we shall not enter in more details.

\section{$3 \quad$ Film geometry and FSC}

In order to clarify the application of the Hamiltonian (11) to FS systems we shall consider simple lattice sums which appear in the one-loop perturbation contributions to the "selfenergy" parameter $r$ and the interaction constant $u$ :

$$
A_{m}(r)=\frac{1}{V_{d}} \sum_{\vec{k}} S_{m}(k, r),
$$


where $m=1,2, \ldots, k=|\vec{k}|, V_{d}=\left(L_{1}, \ldots, L_{d}\right)=V_{D} / L_{0}$, and

$$
S_{m}(k, r)=\frac{1}{L_{0}} \sum_{k_{0}} \frac{1}{\left(k_{0}^{2}+k^{2}+r\right)^{m}} .
$$

In a close vicinity of the critical point $T_{c}$, where $r=\xi^{-2} \sim 0$, the upper cutoff $\Lambda$ for the wave vector $\vec{q}$ can be ignored and the sums (8) and (9) over $\vec{k}$ and $k_{0}$ can be extended to infinity because the essential contributions to these sums are given only by the small wave numbers $\left(0 \sim q^{2} \sim \xi^{-2}\right)$. As we are interested in the critical behaviour in a close vicinity of the critical point, where $\xi \gg a$, the essential contribution to the sums $S_{m}$ and $A_{m}$ will be given by the terms with wave numbers $q_{\nu} \leq \xi$ and therefore we must choose the cutoff $\Lambda \xi>1$. This is consistent with the LWLA provided $(a / \xi)<\gamma \ll 1$. Under the latter conditions the cutoff $\Lambda$ can be kept finite or set infinite without any effect on the results of the summation. As we shall apply the Wilson-Fisher RG method, it seems convenient to keep the cutoff $\Lambda$ for the wave vectors $\vec{k}$ and neglect the upper cutoff for the wave numbers $k_{0}$. It is important to keep in mind that our investigation of the critical behaviour of slabs is valid only for

$$
\xi \Lambda \gg 1, \quad L_{0} \gg a
$$

While the $\vec{k}$-summation in Eq. (8) can be always replaced by a $\vec{k}$-integration, the summation (91) over the wave vector component $k_{0}$ can be transformed to an integration only if certain conditions are satisfied. The latter become clear from the result of the summation (91) over $k_{0} \in(-\infty, \infty)$ for $m=1$ :

$$
S_{1}(k, r)=\frac{L_{0}}{2 y(k)} \operatorname{cth}\left[\frac{y(k)}{2}\right],
$$

where $y(k)=L_{0}\left(k^{2}+r\right)^{1 / 2}$. For $y(k) \ll 1, S_{1}$ from (11) coincides with the $\left(k_{0}=0\right)$-term in (9). In this case, the $D$-dimensional film has a $d=(D-1)$ dimensional behaviour. The quantity $y(k)$ is much less than unity for any $k$ only if $\left(L_{0} \Lambda\right) \ll 1$ and $y(0) \equiv y=$ $\left(L_{0} / \xi\right) \ll 1$. These conditions are consistent with (10) provided $a \ll L_{0} \ll(1 / \Lambda)$. The condition $\left(L_{0} \Lambda\right) \ll 1$ is satisfied in a broad interval of values $L_{0}>a$ because of the fact that $\Lambda \ll(\pi / a)$. Moreover, having in mind that the small values of the wave number $k$ yield the essential contribution in the sums $A_{m}$ one may consider the condition $L_{0} \ll \xi$ instead of $\left(L_{0} \Lambda\right) \ll 1$. This new condition for $y(k) \ll 1$ is weaker than $\left(L_{0} \Lambda\right) \ll 1$. Therefore, the limiting case $y(k) \sim 0$ does not necessarily correspond to an exactly twodimensional $(2 D)$ film (a single atomic layer; $L_{0}=a$ ). Rather, we have shown that the exactly $2 D$ films are beyond the scope of the LWLA and that one of the main limiting cases of our consideration is the quasi- $2 D$ film defined by $a \ll L_{0} \ll(1 / \Lambda)$.

The second limiting case is given by $y(k) \gg 1$. The cases $y(k) \ll 1$ and $y(k) \gg 1$ are often referred to as the limits $y(k) \rightarrow 0$ and $y(k) \rightarrow \infty$, respecticely. For $y(k) \rightarrow \infty$, the Eq. (11) yields a result that can be obtained by replacing the sum $S_{1}$ by an integral 
over $k_{0}$, namely, by taking the continuum limit along the small size $L_{0}$. This corresponds to a $D$-dimensional behaviour of the system when all summations over the wave vector components $q_{\nu}$ can be substituted with an integration over the vector $\vec{q}$. The quantity $y(k)$ tends to infinity for any $k$ only if $y \rightarrow \infty$. Thus our film is quasi-3D for all $L_{0} \gg \xi$.

The behaviour of the sum $S_{1}$ in the two limiting cases $y(k) \rightarrow 0$ and $y(k) \rightarrow \infty$ exhibits the dimensional FSC: $d \rightarrow D=(d+1)$. Having in mind that $A_{m+1}=-\left(\partial A_{m} / \partial r\right)$, $m=1,2, \ldots$, we see that this dimensional crossover is a property of all perturbation terms and, hence of the system as a whole.

The present discussion is particularly important for RG studies of the FSC, where the perturbation integrals are calculated at $r=0$. In such studies the asymptotics of the corresponding integrands are considered and, therefore, one must be sure that the product $k L_{0}$ can reach the limiting values $\left(k L_{0}\right) \ll 1$ and $\left(k L_{0}\right) \gg 1$ within the limitations (10) [8]. The RG integrals are given by taking the continuum limit for the summation over $\vec{k}$ in (8) and by confining the integration in the limits $k \in[\Lambda / b, \Lambda]$, where $b>1$ is the $\mathrm{RG}$ rescaling number. For such integrals with a lower cutoff $\Lambda / b$ the condition $\left(\Lambda L_{0}\right) \gg b$ for thick (almost-3D) films at the critical point $(r=0)$ is satisfied for $L_{0} \gg(b a / \gamma \pi)$. The condition for thin (quasi- $2 D$ ) films is $\left(\Lambda L_{0}\right) \ll 1$ and, together with $a \ll L_{0}$, we have $a \ll L_{0} \ll(a / \gamma \pi)$. All these conditions are consistent within the LWLA and the requirement for a criticality as given by inequalities (10).

Therefore the RG investigations are consistent with both the aims and the model chosen in this paper. The behaviour of the exactly $2 D$ films is beyond the scope of our consideration but we can reliably investigate the FSC from quasi- $3 D$ to quasi- $2 D$ films.

\section{Integration in noninteger dimensionalities}

An obvious disadvantage of all existing descriptions of the FSC and other dimensional crossovers [17] is that the limiting cases can be easily proven and described but the intermediate case $(0<y<1)$ presents a difficult and unresolved task. The systematic way of investigation of the intermediate cases $(y \sim 1)$ is to use the Euler-Maclaurin summation formula and take into account the corrections in inverse powers of $y(k)$ to the continuum limit. Such a treatment requires a numerical analysis. Alternatively, one may perform the RG studies by an integration in noninteger dimensionalities. In this Section we shall discuss the advantages and the disadvantages of this method. We shall show that it can be applied as an interpolation between the limiting cases $y \ll 1$ and $y \gg 1$ only to specific theoretical schemes such as the RG. 


\section{$4.1 \delta$-integration}

Let us consider the integral

$$
A_{1}(r, b)=L_{0} \int \frac{d^{d} k}{(2 \pi)^{d}} \frac{\operatorname{cth}[y(k) / 2]}{2 y(k)},
$$

which follows from (8) and (11). The integrand $S_{1}(k, r)$ exhibits a single-power behaviour $\left[\sim y^{\sigma}(k)\right]$ only for $y(k) \ll 1$ and $y(k) \gg 1$. The existence of a leading power dependence of the integrals $A_{m+1}=-\left(\partial A_{m} / \partial r\right)$ on $y=\left(L_{0} \sqrt{r}\right)$ and the irrelevanve of the correction terms lead to a simple structure of the RG equations and, hence, to their scale invariant solutions, which are important for the description of the critical behaviour. The problem is to achieve such solutions for the intermediate cases of $y \sim 1$, too.

It is impossible to construct an exact integral counterpart of the Eq. (12) with a power law behaviour with respect to $y$ and for this reason, here we shall consider an approximate solution of the problem. We shall substitute the sum $S_{1}(k, r)$ with the $\delta$-dimensional integral

$$
S_{1}^{\prime}=L_{0}^{\delta-1} \int \frac{d^{\delta} x}{(2 \pi)^{\delta}} \frac{1}{\left(x^{2}+k^{2}+r\right)} .
$$

Accordingly, the integral (12) will be substituted by the double $(\delta, d)$-dimensional integral

$$
A_{1}^{\prime}(r, b)=L_{0}^{\delta-1} \int \frac{d^{d} k}{(2 \pi)^{d}} \int \frac{d^{\delta} x}{(2 \pi)^{\delta}} \frac{1}{\left(x^{2}+k^{2}+r\right)} .
$$

The alternative is to substitute the integral (12) with the $(d+\delta)$-dimensional integral

$$
A_{1}^{\prime \prime}(r, b)=L_{0}^{\delta-1} \int \frac{d^{d+\delta} q}{(2 \pi)^{d+\delta}} \frac{1}{\left(q^{2}+r\right)},
$$

where $\delta \in[0,1]$. Certainly, these substitutions are not exact counterparts of the original quantities and their utility in our attempts to present a reliable interpolation between the limiting cases should be justified.

The integral (15) can be deduced after the conjecture that the wave vector component

$k_{0}$ is a $\delta(<1)$-dimensional (sub)vector, $\vec{k}_{0}=\left(k_{\mu 0} ; \mu=1, \ldots, \delta\right)$ and, accordingly, that the total wave vector $\vec{q}=\left\{k_{\mu 0} ; k_{i}\right\}$ and the volume $V_{D}=L^{\delta} V_{d}$ in the sum (8) correspond to a $(d+\delta)$-dimensional system [11, 12, 14]. The integrals $A_{1}^{\prime \prime}$ and $A_{(m+1)}^{\prime \prime}=-\left(\partial A_{m}^{\prime \prime} / \partial r\right)$ have been used in preceding studies of quantum systems [12] and extended impurities [14 for both $\delta>1$ and $\delta<1$.

The integrals $S_{1}^{\prime}$ and $A_{(m+1)}^{\prime}=-\left(\partial A_{m}^{\prime} / \partial r\right)$ given by (13) and (14) are defined with the help of another conjecture, namely, that one may perform a smooth interpolation between the integral values in the continuum limits $(\delta=0)$ and $\delta=1$ for the $d$ - and $D=(d+1)$ dimensional cases, respectively, with the help of the formal rule

$$
\frac{1}{L_{0}^{\delta}} \sum_{k_{0}} \rightarrow \int \frac{d^{\delta} x}{(2 \pi)^{\delta}} \equiv K_{\delta} \int_{0}^{\infty} d x \cdot x^{\delta-1}
$$


where $K_{\delta}=2^{1-\delta} / \pi^{\delta / 2} \Gamma(\delta / 2)$. Using (8) , (9) and (16) one immediately obtains the integrals (13) and (14). The limit $\delta \rightarrow 0$ in the last integral (in spherical coordinates) in (16) should be taken with a special attention because of the divergency of the gamma function $\Gamma(\delta / 2)$. At first one should perform the integration over $x$ of the integrand, say, $x^{+0} f(x) / x$, and then to take the limit $\delta \rightarrow 0$. Usually, the integrands $[\sim f(x)]$ which appear by the perturbation series are such that no divergences arise in the final results for $\delta \sim 0$. This is confirmed by a direct calculation of the integrals (13), (14) and $A_{m+1}^{\prime}=-\left(\partial A_{m}^{\prime} / \partial r\right)$.

For $\delta \rightarrow 0$ and $\delta \rightarrow 1$, the integrals (13) and (14) exactly reproduce the results from (11) and (12) for $y \rightarrow 0$ and $y \rightarrow \infty$, respectively. The same is valid for the integral (15) with respect to Eq. (12). So there are some grounds for the supposition that the intermediate states $(y \sim 1)$ could be interpolated by the values $0<\delta<1$.

\subsection{Effective dimensionality of the fluctuation modes}

The coincidence of the results for the original integrals $A_{m}(r)$ in the limits $y \rightarrow 0$ and $y \rightarrow \infty$ with the results from the integrals $A_{m}^{\prime}$ and $A_{m}^{\prime \prime}$ could be used as a basis of the supposition that there exists a continuous increasing function $\delta(y)$ with the properties $\delta(y \rightarrow 0) \rightarrow 0$ and $\delta(y \rightarrow \infty) \rightarrow 1$. This supposition presents the opportunity to intruduce a new dimensionality - the spatial dimensionality of the fluctuation modes $\phi(\vec{q})$ given by

$$
D_{\text {eff }}(y)=d+\delta(y) .
$$

The Eq. (17) is a straightforward generalization of the known (from previous FS studies [7, 8]) fact that the FS system abruptly changes its $D$-dimensional behaviour to the corresponding $d$-dimensional behaviour when the thickness $L_{0}$ is lowered to values less than $\xi$. According to the model (10), the system is represented by the field $\phi(\vec{x})$. In this respect the notion for the effective length $D_{\text {eff }}$ is not new but in this Section we consider it more explicitly within the generalized form given by Eq. (17). Besides, in Section 5 and 6 we shall introduce an $\tilde{\epsilon}=\left(D_{\text {eff }}-D_{\text {eff }}^{(U)}\right)$-expansion around the upper borderline effective dimensionality $D_{\text {eff }}^{(U)}$. In general, this is a way to describe the RG scaling in terms of the fundamental ratio $y=\left(L_{0} / \xi\right)$. Unfortunately, the present investigation does not give an opportunity to obtain the function $\delta(y)$ and, hence, $D_{\mathrm{eff}}(y)$. The reason is in the pecuriality of the approach based on the $\delta$-integrations introduced in Section 4.1 .

\subsection{Validity}

Although the formal difference in their definitions, the integrals $A_{m}^{\prime}(r)$ and $A_{m}^{\prime \prime}$ lead to the same results in many practical calculations. An example of such a calculation will be presented in this Section; for other examples, see Sections 5 and 6. The problem is that the values of the original sum (11) and integral (12) do not coincide with the values of the 
corresponding integrals in noninteger dimensinalities $(\delta \neq 0)$. Therefore, the application of the integration in noninteger dimensionalities to concrete problems requires a special attention. The relaibility of such applications should be justified for any particular case. Despite the numerous RG studies which have been already performed with the help of the integrals $A_{m}^{\prime \prime}$, the question about the limitations of the corresponding results has not been considered. In order to justify our RG investigations in Sections 5 and 6, we shall consider this problem. Besides, we shall demonstrate the degree of the approximation by compating the original sum $S_{1}(k, y)$ and integral $A_{1}(y)$, denoting them as functions of $y$, with the corresponding integrals $S_{1}^{\prime}(k, y), A_{1}^{\prime}(y)$, and $A_{1}^{\prime \prime}(y)$.

By calculating the integral (13) for $S_{1}^{\prime}$ and by comparing the result with $S_{1}$ from (11) we obtain

$$
\operatorname{th}[y(k) / 2] \leftrightarrow g_{\delta}[y(k)]
$$

with

$$
g_{\delta}[y(k)]=A(\delta)\left[\frac{y(k)}{2}\right]^{1-\delta},
$$

where $A(\delta)=\pi^{\delta / 2} / \Gamma(1-\delta / 2)$; hereafter the symbol " $\leftrightarrow$ " will denote a "comparison" and nothing else. The comparison (18) can be done for any $k<\Lambda$. For $k=0$, we have $y(0)=y$, for $k \xi=1, y(1 / \xi)=\sqrt{2}$, and for a third wave number $k \xi=\sqrt{3}$, which also has an essential contribution in the integrals over $\vec{k}$, we have $y(\sqrt{3} / \xi)=1$. As we shall see our approximations are not precise enough in order to distinguish between the fits of $S_{1}$ and $S_{1}^{\prime}$ for different wave numbers. Besides, there is a strong argument that the most important value of $k$ at which we should make the comparison (18) is $k=0$. This value corresponds to the uniform mode $\phi(\vec{k}=0)$ describing the spontaneous symmetry breaking in the $d$-dimensional subsystem.

By setting $k=0$ in (18) and (19) we have

$$
\operatorname{th}(y / 2) \leftrightarrow A(\delta)\left(\frac{y}{2}\right)^{1-\delta} .
$$

The 1.h.s and the r.h.s. of (20) are depicted in Fig. 1. From one side it is obvious that $S_{1}^{\prime}(0, y)$ is a good approximation to $S_{1}(0, y)$ in quite broad intervals of values of $y$, for example: $0<y(k)<1$ and $y(k)>4$. From the other side, it becomes evident that the values of $\delta$ that give the best fit of $S^{\prime}(0, y)$ to $S(0, y)$ are: $\delta=0$ - for the case $y<1$, and $\delta=1$ - for the case $y \gg 1$. This means that, within the present consideration, the function $\delta(y)$ can be approximated with zero for all $y<1$ corresponding to a relatively good fit of the curves th $(y / 2)$ and $g_{\delta}(y)$ and that, owing to the same arguments, $\delta(y) \approx 1$ for $y \gg 2$. In a broad region of values $y$ around $y=2$ the approximation of $S(0, y) \sim S^{\prime}(0, y)$ breaks down and we could not deduce any reliable conclision about the exact critical value $y_{c}$ of $y$ at which the FSC occurs. According to the present picture one may speculate that this value is probably $y_{c}=2$ whereas the intuitively appealing value is $y=1$. The value $y_{c}=2$ comes from the factor $(1 / 2)$ in the front of $y(k)$ in Eqs. (18), (19), and (20). The picture outlined from the comparison (20) is valid for the comparison denoted in (19). 
In this case one should change $y$ with $y(k)$. For $k=(\sqrt{3} / \xi), y_{c}=1$, and the points of intersection of $g_{\delta}$-lines will be located around the coordinate $y_{c}=1$.

The calculculations within the present approach yield that the locations of the minimal values of the difference between the r.h.s. and the l.h.s. of (20) are $\delta=0$ for all $y<2$ and $\delta=1$ for all $y>1$, namely, that $\delta(y)$ coincides with the $\Theta$-function $\Theta(y-2)$. Certainly this qualitatively wrong result is due to the obvious fact, see Fig. 1, that in a broad region around the point $y_{c}=2$, the approximation of $S_{1}(0, y)$ to $S_{1}^{\prime}(0, y)$ is not valid. It can be however concluded from such rough considerations that the real function $\delta(y)$ will have a steep increase from very small values $\delta \sim 0$ up to $\delta \sim 1$ in a relatively close vicinity $\left(\left|y-y_{c}\right| \sim y_{c}\right)$ of the real critical point $y_{c}$.

The comparison of the integral $A_{1}(r)$ with $A_{1}^{\prime}(r)$ and $A_{1}^{\prime \prime}(r)$ is more difficult. In order to avoid the cutoff $\left(\Lambda^{-}\right)$dependence of the results and simultaneously to avoid irrelevant to our problem ultraviolet divergences we shall set $\Lambda=\infty$ and consider the differences of type $\Delta A_{1}(y)=\left[A_{1}(0)-A_{1}(y)\right]$ rather than the integrals themselves. Using Eq. (12) we obtain

$$
\Delta A_{1}(y)=\frac{1}{2} K_{d} L_{0}^{1-d} I_{d}(y)
$$

with

$$
I_{d}(y)=\int_{0}^{\infty} d z z^{d-1}\left[\frac{\operatorname{cth}(z / 2)}{z}-\frac{\operatorname{cth}\left(\sqrt{z^{2}+y^{2}} / 2\right)}{\sqrt{z^{2}+y^{2}}}\right],
$$

where $z=L_{0} k$. The difference $\Delta A_{1}^{\prime}(y)=\left[A_{1}^{\prime}(0)-A_{1}^{\prime}(y)\right]$ is obtained from Eqs. (8) and (14):

$$
\Delta A_{1}^{\prime}(y)=A(\delta) K_{d} L_{0}^{1-d} J_{d}^{\prime}(\delta, y)
$$

where

$$
J_{d}^{\prime}(\delta, y)=\int_{0}^{\infty} d z z^{d-1}\left[\frac{1}{z^{2-\delta}}-\frac{1}{\left(z^{2}+y^{2}\right)^{1-\delta / 2}}\right] .
$$

Finally, from Eqs. (8) and (15) we have that the difference $\left[A_{1}^{\prime \prime}(0)-A_{1}^{\prime \prime}(y)\right]$ is given by

$$
\Delta A_{1}^{\prime \prime}(y)=K_{d+\delta} L_{0}^{1-d} y^{2} \int_{0}^{\infty} d z \frac{z^{d+\delta-3}}{z^{2}+y^{2}} .
$$

The integrals (22), (24) and (25) have obvious ultraviolet and infrared divergences at the corresponding lower and upper borderline dimensionalities. In order to avoid unnecessary complications in our calculation we shall consider the case of real films $(d=2)$.

For $(d=2)$ the Eqs. (25) becomes

$$
\Delta A_{1}^{\prime \prime}(y)=\frac{A(\delta)}{2 \pi L_{0}}\left(\frac{y^{\delta}}{\delta}\right) .
$$

Because of the obvious infrared divergence at $\delta=0$, we shall consider the derivative $\left(\partial \Delta A_{1}^{\prime \prime} / \partial y\right)$ instead of the difference $\Delta A_{1}^{\prime \prime}$ itself:

$$
\frac{\partial \Delta A_{1}^{\prime \prime}(y)}{\partial y}=\frac{A(\delta) y^{\delta-1}}{2 \pi L_{0}}
$$


The ultraviolet divergences in the two parts of the integral $J_{2}^{\prime}(\delta, y)$ are exactly compensated each other and this integral takes the simple form $J_{2}^{\prime}(\delta, y)=y^{\delta} / \delta$. Thus we obtain that the derivative of $\Delta A_{1}^{\prime}(y)$ is equal to the derivative (27). The integral $\partial I_{2}(y) / \partial y$ can be represented in the form

$$
\frac{\partial I_{2}(y)}{\partial y}=y \int_{y}^{\infty} d t\left(\frac{\operatorname{cth}(t / 2)}{t^{2}}+\frac{1}{2 t \operatorname{sh}^{2}(t / 2)}\right)
$$

which directly yields the result $\operatorname{cth}(y / 2)$. For $d=2$, the derivative of the difference $\Delta A_{1}(y)$ becomes

$$
\frac{\partial A_{1}(y)}{\partial y}=\frac{1}{4 \pi L_{0}} \operatorname{cth}\left(\frac{y}{2}\right)
$$

The correspondence (20) straingtforwardly follows from the comparison of the Eqs. (27) and (29).

Thus we have shown that the derivatives of the integrals $A_{m}^{\prime}$ and $A_{m}^{\prime \prime}$ are quite different the derivatives of the original integrals $A_{m}$, in particular for $y \sim y_{c}$. Having in mind that the values of all integrals coincide at the limiting points $\delta(y=0)=0$ and $\delta(y=\infty)=1$, the same conclusion is true for the integrals themselves.

The demonstrated deviation of the integrals in noninteger dimensionalities from the initial integrals $A_{m}$ does not mean that the RG analysis based on them is unreliable for $0<\delta<1$. The argument here is that the RG predictions about the critical behaviour follow from the RG transformation which reflects the structure and the symmetry of the Hamiltonian rather than from the values of the perturbation integrals. The latters determine the location of the fixed points (FPs) of the RG equations and, therefore, might be of interest only in problems of special interest as to the question of whether a particular stable FP is accessible by the RG flows or not. For such special questions which often arise in investigations of complex systems the wrong determination of the FP coordinates, given by the integrals $A_{m}^{\prime}$ or $A_{m}^{\prime \prime}$, may produce wrong conclusions. Hopefully, our RG analysis in the next two Sections do not come upon such problems and we can arrive at reliable predictions about the critical behaviour of impure films irrespectively on the incorrest predictions about the location of the FPs.

In Sections 5 and 6 we shall perform the RG investigation with the help of the integrals $A_{m}^{\prime}$. This more difficult variant is chosen to demonstrate that the results do not depend on the particular scheme of calculation.

\section{RG analysis: short-range impurity correlations}

The conventional RG treatment of the FSC has been presented in details in preceding works [7, 8, where the perturbation integrals $A_{m}$ have been calculated by a direct summations over $k_{0}$; see, e.g. Eqs. (11) and (12). We shall show that the application of the integration in noninteger dimensionalities yields more information about the critical 
properties of films than the standard RG treatment of FS systems. For this aim we shall use the double $(\delta, d)$-integration.

The RG recursion relations can be derived in two equivalent ways: by introducing an initial rescaling of the wave vector component $k_{0}$ and without such an initial rescaling [7, 8]. We choose the latter variant in which the rescaling of the wave number $k_{0}$ or, equivalently, of the thickness $L_{0} \sim\left(1 / k_{0}\right)$, will appear as a result of the $\mathrm{RG}$ transformation. The initial rescaling transformations are $k_{i}^{\prime}=b k_{i}$ and

$$
\phi_{\alpha}\left(k_{0}, b^{-1} \vec{k}\right)=b^{1-\eta / 2} \phi_{\alpha}^{\prime}\left(k_{0}, \vec{k}\right) \text {, }
$$

where $b>1$ is the rescaling number. In our investigation within the one-loop approximation the Fisher exponent $\eta$ is equal to zero. The calculations are carried out by the direct way 9, 13] of the averaging of the random functions.

Using the standard way of calculations we have derived the RG recursion relations for short-range correlated point impurities (41) in the form

$$
\begin{gathered}
L_{0}^{\prime}=b^{-1} L_{0}, \\
r^{\prime}=b^{2}\left\{r+[4(n+2) u-\Delta] A_{1}^{\prime}(r, b)\right\}, \\
u^{\prime}=b^{3-d}\left\{u-\left[4(n+8) u^{2}-6 u \Delta\right] A_{2}^{\prime}(0, b)\right\}, \\
\Delta^{\prime}=b^{3-d}\left\{\Delta-\left[8(n+2) u \Delta-4 \Delta^{2}\right] A_{2}^{\prime}(0, b)\right\},
\end{gathered}
$$

where the integrals $A_{m}^{\prime}(r, b)$ are the continuum limits of the sums (8) with the upper cutoff $\Lambda=1$ and an lower cutoff $b^{-1}$; hereafter we shall omit the (') of these integrals. The calculation of the integrals to first order in the small parameter $r$ at the upper borderline dimensionality $\left[D_{\mathrm{eff}}^{(U)}=(d+\delta)_{U}=4\right]$ yields:

$$
A_{1}(r, b)=A_{1}(0, b)-A_{2}(0 . b) r
$$

with

$$
A_{1}(0, b)=L_{0}^{\delta-1} \tau(\delta)\left(\frac{1-b^{-2}}{2-\delta}\right)
$$

and

$$
A_{2}(0, b)=L_{0}^{\delta-1} \tau(\delta) \ln b
$$

where

$$
\tau(\delta)=\left(1-\frac{\delta}{2}\right) 2 A(\delta) K_{4-\delta}
$$

Further, we substitute the results (35) - (37) in the relations (32) - (34) and perform the change of the parameters $u$ and $\Delta$ with $\tilde{u}_{0}=L_{0}^{\delta-1} \tau(\delta) u_{0}$ and $\tilde{\Delta}=L_{0}^{\delta-1} \tau(\delta) \Delta$. In the new notations, the Eqs. (32) - (34) take the form

$$
r^{\prime}=b^{2}\left\{r+[4(n+2) \tilde{u}-\tilde{\Delta}]\left(\frac{1-b^{-2}}{2-\delta}-r \ln b\right),\right.
$$




$$
\begin{aligned}
\tilde{u}^{\prime} & =b^{4-d-\delta}\left\{\tilde{u}-\left[4(n+8) \tilde{u}^{2}-6 \tilde{u} \tilde{\Delta}\right] \ln b\right\}, \\
\tilde{\Delta}^{\prime} & =b^{4-d-\delta}\left\{\tilde{\Delta}-\left[8(n+2) \tilde{u} \tilde{\Delta}-4 \tilde{\Delta}^{2}\right] \ln b\right\} .
\end{aligned}
$$

The relation (31) describes the trivial scale invariance of the thickness $L_{0}$. This triviality is a result of the equivalence of the short-range random correlations along all $D$ spatial directions (isotropy of the disorder). As we shall see in Section 6, the extended impurities break this isotropy and the RG relation for $L_{0}$ becomes nontrivial. The structure of Eqs. (39) - (41) is the same as that of the known RG equations for infinite systems [13]. The difference is in the parameter $\delta \neq 0$. We shall see in the next Section that this simple structure of the $\mathrm{RG}$ equations reflects the above mentioned isotropy in the distribution of the disorder. Note, that the factor $1 /(2-\delta)$ in Eq. (39) is relevant only for the precise determination of the FP value of the parameter $r$. The same iz valid for the $\delta$-dependence of the integrals (35) - (37). In Section 4.3 we have already mentioned that the critical behaviour represented by the critical exponents does not depend on the concrete values of the FP coordinates in the parameter space $(r, u, \Delta)$ or, alternatively, in $(r, \tilde{u}, \tilde{\Delta})$.

The $(d, \delta)$-integration is a relatively clumsy variant of calculation and cannot be easily applied in RG calculations in higher loop approximations. But the same results are obtained by the $(d+\delta)$-integration (15) which can be straightforwardly extended to considerations in higer orders of the loop expansion. Thus one can directly obtain the two-loop results for the same problem. The structure of the $R G$ equations is again the same as that for the impurity problem in the corresponding infinite systems [13. The simple difference is that one should change $d$ with $(d+\delta)$, as it is for the Eqs. (39) - (41).

Thus we arrive at the main conclusion that irrespectively to the considered order of the loop expansion, the results for the impure films are obtained from those for infinite systems by the simple substitution of $\epsilon=(4-d)$ with $\tilde{\epsilon}=[4-(d+\delta)]=\left(4-D_{\text {eff }}\right)$. The same is valid for the FP coordinates. Alternatively, by using the $\tilde{\epsilon}$-expansion one obtains the results for both the $D$-dimensional hyperslab $(\delta>0)$ and the corresponding infinite system $(\delta=0)$. For the case of slabs we use the $\tilde{\epsilon}=(d+\delta)$-expansion as shown in Eqs. (39) - (41) and for the infinite system we take the limit $\delta \rightarrow 0$, which means to perform an expansion in $\tilde{\epsilon} \equiv \epsilon=(4-d)=(5-D)$. The reason for this $(5-D)-$ expansion for the infinite system is in the FSC $(d \rightarrow D)$ denoting the equivalence between the critical behaviour in $D$-impure films and that in $d=(D-1)$-dimensional impure infinite systems. Alternatively, in case of infinite systems, one may take the limit $\delta \rightarrow 1$, $D_{\text {eff }}=(d+\delta) \rightarrow D$ and, therefore, the expansion parameter is $\epsilon=4-D$.

Within the present RG analysis we have shown that the FSC can be described as a smooth change of the behaviour of the system with the variation of $\delta$ from $\delta=0$ to $\delta \rightarrow 1$. Bearing in mind our discussion in Section 4, the variations of $\delta$ between these limiting values mean a corresponding variation of the ratio $y=\left(L_{0} / \xi\right)$ from $y \ll 1$ to $y \gg 1$. Although the smooth variation of $\delta$ in the RG equations in the broad interval $(0,1)$, the FS crossover is expected to occur mainly in the interval $\left|y-y_{c}\right| \sim y_{c}$, where the $y_{c} \sim 1$ is the critical ratio. 
In general, the critical behaviour of the hyperslab will correspond to the MF description for geometrical dimensionalities $D>5-\delta$. As our RG results are valid above the lower effective borderline dimensionality $(d+\delta)_{L}=2$, the nontrivial impure (for $n<4$ ) or pure (for $n>4$ ) stable critical behaviour will occur for geometrical dimensionalities $(3-\delta)<D<(5-\delta)$. We shall not derive and discuss the FPs and the related critical and stability exponents of the impure films because we have demonstrated that these quantities and, therefore, the critical behaviour of the film as a whole, are obtained by setting $\epsilon=(4-d) \rightarrow \tilde{\epsilon}=(\epsilon-\delta)$ in the known results for the respective quantities of impure infinite systems with the same type of disorder [13].

For real slabs $(D=3)$ we find that the nontrivial critical behaviour will appear for $\delta>0$. This implies $L_{0} \gg a$ (quasi-2D films) and, moreover, the real films should be thick enough to ensure a parameter $\delta>0$. In view of the discussion in Section 4 , this probably corresponds to the ratio $\left(L_{0} / \xi\right)$ of order of the unity. Denoting $\tilde{\epsilon}=(\epsilon-\delta)$, where $\epsilon=(4-d)$, we see that the results initially obtained for $\tilde{\epsilon} \ll 1$ can be extrapolated to real films $(d=2)$ provided we extend the values of our small parameter to $1<\tilde{\epsilon}=(2-\delta)<2$. This is an advantage with respect to the simple extension of $\epsilon=(4-d)$ to $\epsilon=2$ for $2 D$ films. There are no problems for the extrapolations of the $\tilde{\epsilon}$-results because the RG analysis does not demonstrate any peculiarities of the RG analysis at these relatively low dimensionalities such as: the appearance of new FPs, any runway of the knoun $($ at $\tilde{\epsilon} \ll 1)$ FPs, a qualitative change of the stability properties of the FPs or, a change of the location of the FPs in domains of the parameter space, where they could be unaccessible for the RG flows.

From one side, we have shown, that the scope of the RG investigations with the help of field theretical models does not include $2 D$-dimensional films (mono-layers) or extremely thin films consisting of several atomic layers $\left(L_{0} \sim a\right)$. From the other side, it has been completely clarified that the present approach can be undoubtedly applied to real films $(D=3)$.

\section{$6 \quad$ RG for extended impurities}

\subsection{RG equations}

The RG equations for the case the extended impurities and inhomogeneities described by the Eqs. (5) -(17) are derived in the way outlined in the preceding Section. In order to simplify the notations we introduce the new variables $v=L_{0}^{\delta-1} \tau(\delta) u$ and $\mu=K_{4-\delta} \Delta$. Then, the RG relations can be written in the form

$$
k_{0}^{\prime}=b k_{0}\left\{1+\mu \frac{b^{\delta}-1}{2 \delta}\right\},
$$




$$
\begin{gathered}
r^{\prime}=b^{2}\left\{r+4(n+2) v\left[\frac{1-b^{-2}}{2-\delta}-r \ln b\right]\right. \\
\left.-\mu\left[\frac{1-b^{-2+\delta}}{2-\delta}-r\left(\frac{b^{\delta}-1}{\delta}\right)\right]\right\}, \\
v^{\prime}=b^{4-d}\left(\frac{L_{0}^{\prime}}{L_{0}}\right)^{\delta}\left\{v-4(n+8) v^{2} \ln b+6 v \mu\left(\frac{b^{\delta}-1}{\delta}\right)\right\}, \\
\mu^{\prime}=b^{4-d}\left\{\mu-8(n+2) v \mu \ln b+4 \mu^{2}\left(\frac{b^{\delta}-1}{\delta}\right)\right\} .
\end{gathered}
$$

These RG relations have been obtained by the $\epsilon=\left(4-D_{\text {eff }}\right)$-expansion around the upper borderline dimensionality $D_{\mathrm{eff}}^{(U)}=4$ (see Section 6 ). In contrast to the case in Section 6 the scaling invariant solutions of these RG relations cannot be found for any $\delta \in[0,1]$. In order to obtain such solutions we must consider $\delta \ll 1$ and to substitute the factor $\left(b^{\delta}-1\right) / \delta$ with $\ln b$. As our expansion parameter is $\tilde{\epsilon}=[4-(d+\delta)] \ll 1$, this means that we must investigate the case when $\delta \ll$ and $\epsilon=(4-d) \ll 1$. Thus we have a single $\tilde{\epsilon}-$ expansion with two small parameters: $\epsilon=(4-d)$ and $\delta$. This variant of the theory is often reffered to as a "double $(\delta, \epsilon)$-expansion" [10, 14]. The anisotropy of the random correlations "breaks" the single $\tilde{\epsilon}$-expansion to a double one.

The $\mu$-contribution in the RG relation (30) for $k_{0}$ comes from a perturbation term shown diagrammatically in Fig. 2. In contast to the usual one-loop results, this self-energy contribution depends on the wave number $k_{0}$. The reason is that the broken line of the diagram in Fig. 3 does not carry external wave numbers $k_{0}$. This is a direct consequence of the fact that the random function $\varphi(\vec{k})$ does not depend on $k_{0}$, i. e. of the infinite ranged correlations along the small site $L_{0}$. The $\mu$-dependence in the RG equation for $k_{0}$ leads to a nontrivial scaling relation for the thickness $L_{0}$, namely,

$$
L_{0}=L_{0}^{\prime} b^{1+\mu / 2}
$$

which is quite similar to the scaling relations for the temperature $T$ known from studies of quantum critical phenomena [17. The scaling law (34) has the critical exponent

$$
z_{d}=1+\frac{\mu^{*}}{2}
$$

where the asterisk $(*)$ denotes any $\mathrm{FP}$ value of $\mu$. This exponent is analogous to the dynamical critical exponent in the theory of dynamical critical phenomena in classical models with quenched disorder [15] and quantum systems [16, 17].

Using the relation (46) and $\delta \ll 1$, the Eqs. (43)-(45) can be written in the form

$$
\begin{aligned}
& r^{\prime}=b^{2}\left\{r+[4(n+2) v-\mu]\left[\frac{1-b^{-2}}{2}-r \ln b\right]\right\}, \\
& v^{\prime}=b^{4-d-\delta(1+\mu / 2)}\left\{v_{0}-\left[4(n+8) v^{2}-6 v \mu\right] \ln b\right\}
\end{aligned}
$$




$$
\mu^{\prime}=b^{4-d}\left\{\mu-\left[8(n+2) v \mu-4 \mu^{2}\right] \ln b\right\} .
$$

The extra-factor $b^{-\delta \mu / 2}$ in Eq. (49) is not essential for the RG analysis to this $\left(\tilde{\epsilon}^{1-}\right)$ order of the theory because $\mu \delta \sim \epsilon \delta \sim \epsilon^{2}$.

The RG relations (48)-(50) yield new $\delta$-corrections to the relevant physical quantities. They present the difference between the critical properties of the impure film and the corresponding infinite impure system.

\subsection{FPs and critical exponents}

The RG relations (48) - (50) have four FPs: the Gaussian FP (hereafter referred to as GFP) with coordinates $\left(u_{G}=\mu_{G}=0\right)$, the so-called "unphysical" FP (UFP), $v_{U}=0$, $\mu_{U}=-\epsilon / 4$, the Heisenberg FP (HFP),

$$
v_{H}=\frac{\epsilon-\delta}{4(n+8)}, \quad \quad \mu_{H}=0,
$$

and the random FP (RFP),

$$
v_{R}=\frac{\epsilon+2 \delta}{16(n-1)}, \quad \mu_{R}=\frac{(4-n) \epsilon+2(n+2) \delta}{8(n-1)} .
$$

All these FPs are known by the work of T. C. Lubensky [13]. Here the FPs require a further investigation because of the FS effect $(\delta>0)$.

As usual, the GFP always exists and is stable for $d>4$. The UFP has no physical meaning for $\epsilon>0$, namely, for $d<4$, because the parameter $\mu$ must always be nonnegative $(\mu \sim \Delta)$. For $d>4$, the UFP is physical $\left(\mu_{U}>0\right)$ but unstable. For $4<d<(4+2 \delta)$, the UFP has an instability towards $\mu$, whereas the same FP has a double instability (towards both $v$ and $\mu$ ) for $d>(4+2 \delta)$. Further, we shall concentrate our attention on the GFP, HFP and RFP. In order to analyze their properties we must obtain the critical and stability exponents.

The stability exponents of the GFP are $\lambda_{v}^{(G)}=\epsilon-\delta$ and $\lambda_{\mu}^{(G)}=\epsilon$. These values show that the GFP is stable only for $d>4$, where it describes an usual (free of disorder and fluctuation interactions) Gaussian behaviour. The stability exponents of the HFP are

$$
\lambda_{v}^{(H)}=\delta-\epsilon,
$$

and

$$
\lambda_{\mu}^{(H)}=\frac{(4-n) \epsilon+2(n+2) \delta}{(n+8)} .
$$

The stability exponents $\lambda_{1,2}^{(R)}$ of the RFP are given by

$$
\lambda_{1,2}^{(R)}=-\frac{1}{8(n-1)}[3 n \epsilon+2(4-n) \delta \mp \sqrt{\Theta}]
$$


where

$$
\begin{aligned}
\Theta & =(5 n-8)^{2} \epsilon^{2}-4\left(15 n^{2}+24 n-48\right) \delta^{2} \\
& -12\left(n^{2}+12 n-16\right) \epsilon \delta .
\end{aligned}
$$

The static critical exponents describing the critical behaviour of the system are given by $\eta=0$ and the value of the correlation length exponent $\nu$. For the GFP we have $\nu_{G}=1 / 2$, for HFP,

$$
\nu_{H}=\frac{1}{2}+\frac{(n+2)}{4(n+8)}(\epsilon-\delta)
$$

and for the RFP,

$$
\nu_{R}=\frac{1}{2}+\frac{3 n \epsilon+2(n+2) \delta}{32(n-1)} .
$$

For $\delta=0$ and, hence, $D_{\text {eff }}=d$, the Eqs. (53)-(58) yield the familiar results for $d-$ dimensional infinite impure systems [13]. For the GFP and HFP we have $z_{d}=1$, whereas for the UFP and RFP $z \neq 1$. For the RFP Egs. (47) and (152) yield

$$
z_{d}^{(R)}=1+\frac{(4-n) \epsilon+2(n+2) \delta}{16(n-1)} .
$$

\subsection{Stability properties of the FPs}

Consider the stabilyty properties of the HFP. The requirement of stability $\lambda_{\mu}^{(H)}<0$ leads to the following inequality

$$
4(4-d+\delta)<(4-d-2 \delta) n
$$

When we solve this inequality with respect to $n$ we should have in mind that the quantities $(4-d)=\epsilon \sim \delta$ are small and that is why it is not convenient to divide the ineq. (60) by the small factor $(4-d-2 \delta)$. For this reason, let us consider first the case $\delta=0$, where the inequality (60) is valid for $n>4$, provided $d<4$. Further, we consider $n=4$ and for this value we easily find that the inequality (60) cannot be satisfied for any $\delta \geq 0$. Therefore, for $n=4$, the HFP is unstable towards the parameter $\mu$, i. e. towards the disorder effects. For infinite systems $(\delta=0)$ with $n=4$, the HFP has a marginal stability $\left(\lambda_{\mu}^{(H)}=0\right)$. The problem of whether the HFP is stable for $n=4$ and $\delta=0$ has been widely discussed in preceding studies [13] in high orders in $\epsilon$. In particular, this topic has been extensively investigated by I. D. Lawrie et al (see Ref.[12]) for the case of impure systems with a cubic anisotropy. It has been shown that the higher orders in the loop expansion do not give the initially expected reliability in the treatment of the stability properties of the HFP near and at the value $n=4$ of the symmetry index $n$. The reason is not in the specific features of the model but is a general disadvantage of the RG studies of systems with competing effects. Therefore, the same problem cannot be reliably solved in the present case, too. 
The inequality (601) is used together with $\lambda_{v}^{(H)}<0$ and $d>(2-\delta)$ in order to demonstrate that the HFP is unstable for all $n<4$. For $n>4$, the stability requirements $\lambda_{v}^{(H)}<0$, $\lambda_{\mu}^{(H)}<0$ and $d>(2-\delta)$ yield the domain of stability

$$
2-\delta<d<d_{H}
$$

where

$$
d_{H}=4-\frac{2(n+2)}{(n-4)} \delta .
$$

In fact, the conditions (61) are satisfied for all $\delta \in[0,1]$ provided $n>16$, and for $0 \leq \delta<\delta_{0}$ with $\delta_{0}=2(n-4) /(n+8)$ provided $4<n<16$. Increasing the value of $\delta$, the interval of variations of $d$ decreases and for $\delta=\max \left(\delta_{H}, 1\right)$ the width of the $d$-domain of stability becomes zero.

The stability properties of the RFP can be investigated only for $n \neq 1$, because of the degeneration [13] of the RG equations at $n=1$. This degeneration leads to a special critical behaviour described by complex stability exponents [13] and, hance, by oscillatory corrections to the main scaling laws [13]. The investigation of the "oscillatory" critical behaviour is made in higher orders of $\epsilon$ and $\delta$. The values $0<n<1$ are of an academic interest only. We shall consider the case $n>1$.

Using the physical requirement $\mu_{R}>0$, one obtains an inequality inverse to (60). Further, we must distinguish between two cases of stability of the RFP: (R) real negative exponents $\lambda_{1,2}^{(R)}<0$, and $(\mathrm{C})$ complex exponets $\lambda_{1,2}^{(R)}$ with negative real parts. For the case (R), using Eqs. (55) - (566), the requirement $\lambda_{1,2}^{(R)}<0, d>(2-\delta)$ and the inequality inverse to (60), we determine the following domain $R_{R}$ of stability in the $(d, n, \delta)$ space:

$$
\begin{gathered}
2-\delta<d<(4+2 \delta), \\
(4-n)(4-d)+2(n+2) \delta>0, \\
3 n(4-d)+2(4-n) \delta>0,
\end{gathered}
$$

and $\Theta \geq 0$. For the case (ii) of complex stability exponents $\lambda_{1,2}^{(R)}$, the domain of stability $R_{C}$ is defined by the inequalities (64), (65), $\Theta<0$, and $d>(2-\delta)$.

The criteria of stability can be investigated numerically for all allowed values of $d, \delta$ and $n \neq 1$. Here we shall restrict the analysis to those values of $n$ which might be of importance for real systems or for the explanation of the properties of the model. The most important theoretical problem is the comparison of the domains of stabilities of the G, H and R FPS for values of $n=2,3,4, \ldots$

For $n=4$, one easily obtains the picture in Fig. 3. The RFP with real exponents $\lambda_{1,2}^{(R)}$ is stable in the domain $R_{R}$ defined by $0<\delta<\delta_{4}$ and $(2-d)<d<d_{R}(4)$, where 
$\delta_{4}=2 /(1+2 \sqrt{3})$ and $d_{R}(4)=4-2(1+\sqrt{3})$. In Fig. 3, the point $a$ has coordinates $\left(\delta_{a} \equiv \delta_{4} \approx 0.45, d_{a}=1.55\right)$, the point $b$ has coordinates $(1,1)$, and the point $c$ is given by $(1,4)$. The domain $R_{C}$ in Fig. 3 denotes the stability region of the random critical behaviour with complex stability exponents. This domain is given by the inequalities $d_{R}(4)<d<4$ provided $\delta<\delta_{4}$, and by $(2-\delta)<d<4$ for $\delta_{4}<\delta \leq 1$.

For $1<n<4$ the stability criteria for $R$ in the case of real stability exponents are $\Theta \geq 0$ and

$$
2-\delta<d<d_{R}
$$

where

$$
d_{R}=4+\frac{2(4-n) \delta}{3 n} .
$$

The domain $R_{C}$ of complex stability exponents is given by the inequalities $\Theta<0$ and (66).

The domains of stability $R_{R}$ and $R_{C}$ for $1<n<4$ are quite similar to those in the case $n=4$ depicted in Fig. 3, but the coordinates of the points $a$ and $c$ in the $(\delta, d)$ plane vary with $n$. The coordinates $\left(\delta_{a}, d_{a}\right)$ and $\left(d_{c}, \delta_{c}=1\right)$ of the points $a$ and $c$ are given in Table 1 for several values of $n$. The value $\delta_{a}$ decreases from 0.45 to zero with the decrease of $n$ from $n \sim 4$ to $n \sim 8 / 5$. For $n<8 / 5, \delta_{a}$ increases with the decrease of $n$.

TABLE 1 . Values of $\delta_{a}, d_{a}$, and $d_{c}$

\begin{tabular}{|l|l|l|l|l|l|l|}
\hline \hline$n$ & $7 / 5$ & $8 / 5$ & $9 / 5$ & 2 & 3 & 4 \\
\hline$\delta_{a}$ & 1.06 & - & 0.02 & 0.05 & 0.26 & 0.45 \\
\hline$d_{a}$ & 1.94 & - & 1.98 & 1.95 & 1.74 & 1.55 \\
\hline$d_{c}$ & 5.24 & 5 & 4.81 & 4.67 & 4.22 & 4 \\
\hline \hline
\end{tabular}

The value $n=8 / 5$ is quite special because for this value the function $\Theta(n, \epsilon, \delta)$ given by Eq. (566) depends linearly on $\epsilon$. Within our one-loop approximation, the domain $R_{R}$ at $n=8 / 5$ does not exist at all and the domain $R_{C}$ is expanded up to $0 \leq \delta \leq 1$ and $2-\delta \leq d \leq 4+\delta$. In our case, the effective values of $\delta_{a}(8 / 5)$ and $d_{a}(8 / 5)$ are 0 and 2 , respectively. It has been shown by I. D. Lawrie et al 13 that the same value $n=8 / 5$ is the reason for the peculiar critical behaviour of infinite impure systems $(\delta=0)$ with a cubic anisotropy. So, one may expect that in our case a similar peculiar behaviour at $n=8 / 5$ with singularities of the $\epsilon-$ expansion and complex exponents will occur.

It is seen from Table 1 that the coordinate $d_{c}(n)$ in Fig. 3 is larger than $d_{c}(4)=4$ for all $0<n<4$. This means that for all $1<n<4$, there is a triangle domain for certain values $d>4$, where both the RFP and the GFP are stable. In this region there is a competition between the pure Gaussian behaviour represented by the GFP and the impure behaviour represented by RFP. The outcome of this competition should depend on the strength of the disorder effect.

For $n>4$, both the RFP and the HFP are stable for certain values of $d$ and $\delta$. The stability domains $R_{R}$ and $R_{C}$ can be determined with the help of the inequalities (63)- 
(65). For $\delta<\delta_{0}$, the domain $R_{R}$ is confined by the inequalities $\Theta \geq 0$, and

$$
d_{H}<d<d_{R}
$$

whereas for $\delta>\delta_{0}$, the same domain is described by the inequalities $\Theta \geq 0$ and

$$
2-\delta<d<d_{R}
$$

The domain $R_{C}$ is defined by the same inequalities (68) and (69) but for $\Theta<0$.

A typical picture of the stability domains in this case is given in Fig. 4 for $n=6$, where the points $h$ and $a$ have coordinates: $\delta_{h} \equiv \delta_{0}(6)=0.29, d_{h}=1.61, \delta_{a}=0.74$ and $d_{a}=1.26$. The HFP is stable for relatively small values of $\delta$, whereas the stability of the RFP dominates for large values of $\delta$. There is a domain $(4-0.22 \delta)<d<4$ of dimensionalities $d \sim 4$ for which both the RFP and the HFP are unstable. This domain is defined by the location of the point $c$ with coordinates $\delta_{c}=1, d_{c}(6)=3.78$; see the shaded region in Fig. 4. Such domains of instability exist for all symmetry indices $n>4$ and $\delta>0$. They can be described by the inequality $d^{\prime}(n, \delta)<d<4$ which can be obtained by the RG analysis. For $n=6$ and $\delta=1, d^{\prime}(n, \delta)=d_{c}(6)$ as shown in Fig. 4. For $\delta \rightarrow 0$, $d^{\prime}(n, \delta) \rightarrow 4$ and for $\delta \rightarrow 1, d^{\prime}(n, \delta)$ is lowered up to a minimal value $d^{\prime}(\infty, 1)=3.33$ (See also Fig. 5).

As the GFP is stable only for $d>4$, the shaded domain in Fig. 5 remains the region of a total instability of the system. This total lack of stable FPs for all $n>4, \delta>0$ and certain $d \sim 4$ can be interpreted as an indication for a fluctuation-driven impure tricritical phenomenon followed by a first-order phase transition. The FS system $(\delta>0)$ is unstable towards the disorder (the parameter $\mu$ ) and, hence, the reason for the appearance of a fluctuation-driven phase transition of first-order is in the simultaneous effect of the FS and the extended impurities.

In order to justify the prediction for the existence of a tricritical point and a fluctuation (or disorder)-driven phase transition of first order, we remind that the fluctuation interactions represented by the parameter $u$ are relatively small near four dimensionalities $(d \sim 4)$. Under such circumstances, the disorder effects may alter the sign of the effectivelly small parameter $u$ from $u>0$ (a second-order transition) to $u<0$ (a first-order transition). At $u=0$, a tricritical point should appear. Note, that these effects are impossible for infinite impure systems and in pure FS systems. The change of the order of the phase transition in the present case is a result of the competition between fluctuation, disorder and FS effects. The effective fluctuation model for the description of this change of the order of the phase transition can be constructed by adding an additional $u_{6} \phi^{6}$ term to the Hamiltonian (11).

The stability domain of the HFP vanishes when the symmetry index $n$ decreases from 6 to 4 . Increasing the number $n$ to $n=16$, the stability region of the HFP expands up to the triangle $2 b 4$ (see the dotted line $4 b$ in Fig. 4). The further increase of the values of $n$ above $n=16$ leads to an enlargement of the stability region of the HFP so that the points 
$h$ and $a$ already lay on the vertical line $\delta=1$. For $n \rightarrow \infty$ (the limit of the spherical model [9]) the point $h$ has coordinates $(1,2)$ on the vertical line $\delta=1$, as shown in Fig. 5 . The $R_{R}$ is extremely small but persists (see the narrow wing $h 4 a$ with $d_{a}=2.19$ ). The domain $R_{C}$ is shown in Fig. 5 by the triangle $a 4 c$, where $d_{c}=3.33$. In Figs. $4-6$, the point $b$ has the same coordinates $(1,1)$.

\section{Summary and validity of the results}

We have obtained and analyzed in details the RG recursion relations for FS systems of slab geometry described by the $n$-component LG model containing quenched impurities. Two models of quenched impurities have been considered. The results demonstrate the outcome of the competition between the effects of fluctuation interactions, the finite size, and the disorder.

We have used an $\tilde{\epsilon}=\left(4-D_{\text {eff }}\right)$-expansion defined by the effective spatial dimensionality $\delta=\left(D_{\text {eff }}-d\right)$ of the fluctuation modes of the order parameter. Our approach is based on an approximate substitution of the lattice summation with an integration in dimensionalities less than unity. The limitations of this approach have been considered. The approach is more general that the familiar RG considerations based on the $\epsilon=(4-d)-$ expansion. The fundamental relationship between the effective dimensionality $D_{\text {eff }}$ and the ratio $y=\left(L_{0} / \xi\right)$ of the characteristic lengths of the film has been introduced and discussed.

The most interesting cases of critical phenomena are those for $\delta \ll 1$ (thin films) and $\delta \gg 1$ (thick films, when the film behaves as an almost-infinite system). It has been demonstrated that our approach based on an integration in dimensionalities less than unity is reliable for the description of these cases, in particular, for real films $(D=3)$.

The method introduced in this paper has been used to demostrate the validity of the FSC in films with homogeneously distributed quenched impurities with short-range random correlations. It has been shown that the proof of the FSC in these systems can be easily proven and straightforwardly expanded to any order in the loop expansion. The reason is that the impurity correlations are spatially isotropic and the sum $(\epsilon+\delta)$ can be taken as the single expansion parameter $\tilde{\epsilon}$.

The extended impurities break the spatial isotropy of the disordered system, and the RG investigation yields a dependence of the critical exponents on two small parameters: $\epsilon=(4-d)=(5-D)$ and $\delta$. This leads to another picture of the critical behaviour which will be summarized in the remainder of this Section.

The dependence of the FP coordinates and the critical exponents on the small parameters $(\epsilon, \delta)$ and the symmetry index $n$ has been calculated and analyzed with respect to the stability properties of the FPs. In this way we have established the possible types of critical behaviour for different values of $\delta$, the geometric dimensionality $D$ of the hyperslab, and 
$n$. In order to describe the random critical behaviour in films with extended impuirities we have introduce and calculated a new critical exponent $z_{d}$. The analogy between this exponent and the dynamical exponent in quantum systems has been emphasized. The results are valid for effective dimensionalities $D_{\text {eff }}>D_{\text {eff }}^{(L)}=2$ - the lower borderline dimensionality.

For real films $(D=3)$ with extended impurities our analysis is valid for the so-called quasi-2D (thin films), where $\delta>0$. So, the results from our RG analysis performed for small values of $\epsilon$ and $\delta$ can be exptrapolated for predictions of the critical behaviour of real impure films $(D=3, d=2)$ with a finine thickness $L_{0} \gg a$, where the $\delta(y)>0$. We have shown that, in the strict mathematical sense, the latter condition is always valid for $y>0$, i. e. when the system is outside the critical point. But in real expriments the conditions for the validity of our consideration are somewhat different. The function $\delta(y) \sim 0$ up to values of $y \sim 1$ and, therefore, in experimental conditions $(\xi \gg a)$ the thickness $L_{0} \sim \xi$ should be much larger than the interparticle distance $a$. As far as the behaviour in the critical region $(\xi \gg a)$ is concerned the condition $y>0$ will be easily satisfied for films with $L_{0} \gg a$. This is the case of interest for experiments. The case of exactly $2 D$ films $\left(L_{0}=a\right)$ corresponds to the lower borderline dimensionality and, therefore, is beyond the scope of our investigation. The invalidity of the results at the lower borderline dimensionality is a generic disadvantage of the field-theoretical RG methods rather than a result of our particular approach to the problem. The $2 D$ case can be effectively achieved for any film in the asymptotic vicinity of the critical point, where $\xi \rightarrow \infty$ and, hence, the ratio $y \rightarrow 0$ for any finite thickness $L_{0}$. However, this situation is not of interest for the most part of real experiments.

While the condition $D_{\text {eff }}>D_{\text {eff }}^{(L)}=2$ guarantees the reliability of the qualitative predictions of our RG analysis, the extrapolation of the results obtained near the upper borderline dimensionality $D_{\mathrm{eff}}^{(U)}=(4-d-\delta)$ to the real dimensionality $(d=2)$ requires an extension of the values of the small parameter $\tilde{\epsilon}$ to values $\tilde{\epsilon} \sim(2-\delta)<2$. This is the usual way of extrapolation of the RG results to real systems. It is believed the small parameter $\tilde{\epsilon}$ should be set equal to $(2-\delta)$ in order to predict the values of the critical exponents for the real case $(\delta \ll 1)$ and that in this case, the one-loop results do not give quantitatively correct predictions. Although this is generally true, the qualitative predictions from the one-loop results are reliable, in particular, for the solution of important problems like the possible types of critical behaviour and the conditions under which a critical behaviour can occur. In this article we have been mainly involved in these type of problems and the results are well grounded within the one-loop approximation. The practice of the numerous applications of the RG to complex systems shows that the one-loop results about the general picture of the critical phenomena remain valid within the framework of consideration by higher loop approximations. For real films $\tilde{\epsilon}=(2-\delta)$ should take values $\tilde{\epsilon} \sim 2$ for $\delta \ll 1$ and $\tilde{\epsilon} \sim 1$ for $\delta \sim 1$ (thick films). Therefore, the analysis carried out in Section 6 is reliable for the case of real films and their critical behaviour is given by Figs. $3-5$ on the line $d=2$. In addition to this conventional point of view 
we shall stress that the results for small values of $\epsilon=(4-d)$ can also be extrapolated to real films but for those parts of the critical region which are not asymptotically close to the critical point. Thus the fluctuation-driven change of the order of the phase transition near the upper borderline dimensionality $(4+\delta)$ can be extrapolated to real effective dimensionality $(d+\delta)$, provided one is interested in fluctuation phenomena which occur outside the asymptotic vicinity of the critical point.

A brief summary of other results for the critical behaviour in real dimensionalities $(d=$ $2,0<\delta<1$ ) can be outlined with the help of Figs. $3-5$. This critical behaviour is described by the HFP and the RFP. For $1<n \leq 4$, only the random critical behaviour is present. For relatively thick films this critical behaviour will exhibit oscilatory corrections to the scaling laws described by complex stability exponents, whereas the ramiliar random critical behaviour will occur in relatively thin films. For $n>4$, the pure critical behaviour represented by the properties of the HFP will occur for relativelu thin films whereas the random critical behaviour in its two variants of real and complex stability exponents will occur in thick films. In the spherical limit $(n=\infty)$ the pure critical behaviour is stable for all real films and the disorder effects are irrelevant for the critical properties. The singular behaviour of the films for the symmetry indices $n=1$ and $n=8 / 5$ has been explained in Section 6 .

Finally, we note, that the present investigation reveals essential differences in the critical behaviour of infinite and FS systems with extended impurities. The finite thickness $L_{0}$ of the film leads to: an essential dependence of the critical exponents on the noninteger dimensional portion $\delta$, a fluctuation-driven change of the order of the phase transition for all $n>4$ and $d \sim 4$ (the almost-MF region), the competition between the pure (Gaussian) and impure critical regimes for all $1<n<4$ and $2 \leq d<4$, and the dominating role of the impure (random) critical behaviour with oscillatory corrections to the scaling laws for all relatively thick films.

\section{Acknowledgments:}

L. Craco and D. I. Uzunov gratefully acknowledge the hospitality of the MPI-PKS (Dresden), Aussenstelle Stuttgart and, in particular, the kind support by Prof. Peter Fulde. L. Craco thanks the support by the Brazilian Agency Conselho Nacional de Desenvolvimento Científico e Tecnológico $(\mathrm{CNPq})$. A research grant Ph560 of NFS (Sofia) is also acknowledged. 


\section{References}

[1] See, e. g., K. Binder, in: Phase Transitions and Critical Phenomena, Vol. 8, ed. by C. Domb and J. L. Lebowitz (Academic Press, London, 1983) p. 1; H. W. Diehl, ibid, Vol. 10, p. 76 .

[2] J. J. Pearson, Phys. Rev. 138 (1965) A213; D. J. Mills, Phys. Rev. B 3 (1971) 3887; R. A. Weiner, Phys. Rev. B 8, (1973) 4427; R. Pandit and M. Wortis, Phys. Rev. B 25 (1982) 3226.

[3] D. I. Uzunov and M. Suzuki, Physica A 204 (1994) 702.

[4] M. I. Kaganov and A. N. Omel'yanchuk, Zh. Eksp. Teor. Fiz. 61 (1971) 1679 [Sov. Phys. - JETP 34 (1972) 895]; T. C. Lubensky and M. H. Rubin, Phys. Rev. B 12 (1975) 3885.

[5] M. E. Fisher, in Critical Phenomena, Proceedings of the 51th Enrico Fermi Summer School, Varenna, Italy; ed. by M. S. Green (Academic Press, New York, 1971) p. 1; M. E. Fisher and M. N. Barder, Phys. Rev. Lett 28 (1972) 1512;

[6] For a review, see, e.g., M. N. Barber, in: Phase Transitions and Critical Phenomena, Vol. 8, ed. by C. Domb and J. L. Lebowitz (Academic Press, London, 1983) p. 145.

[7] See, e.g., J. A. Bray and M. A. Moore, J. Phys. A 11 (1978) 715; I. D. Lawrie, J. Phys. C 11 (1978) 3857; A. M. Nemirovsky and K. F. Freed, J. Phys. A 19 (1986) 591; H. Guo and D. Jasnow, Phys. Rev. B 35 (1987) 1846; D. O'Conner and C. R. Stephens, Nucl. Phys. B 360 (1991) 297.

[8] D. I. Uzunov and M. Suzuki, Phisica A 216 (1995) 489.

[9] D. I. Uzunov, Introduction to the Theory of Critical Phenomena (World Scientific, Singapore, 1993).

[10] See, e.g., G. Grinstein, in Fundamental Problems in Statistical Mechanics VI, ed. by E. G. D. Cohen (North-Holland, Amsterdam, 1985) p. 147; M. McCoy and T. T. Wu, Phys. Rev. Lett. 21 (1968) 549; Phys. Rev. 176 (1968) 631; 188 (1969) 982; 188 (1969) 1014.

[11] L. De Cesare, L. Craco, I. Rabuffo, and D. I. Uzunov, J. Phys. Studies (1998), in press.

[12] A. M. J. Schakel, Phys. Lett. A 224 (1997) 287; T. R. Kirkpatrick and D. Belitz, Phis Rev. Lett 76 (1996) 2571.

[13] T. C. Lubensky, Phys. Rev. B 11 (1975) 3573; D. E. Khmelnitskii, Phys. Lett 67A (1978) 59; I. D. Lawrie, Y. T. Millev and D. I. Uzunov, J. Phys. A 20 (1987) 1599; See also S. Ma, Modern Theory of Critical Phenomena (Benjamin, London 1976). 
[14] S. N. Dorogovtsev, Phys. Lett. 76A (1980) 169; D. Boyanovsky and J. L. Cardy, Phys. Rev. B 26 (1982) 154; 27 (1983) 6971(E); I. D. Lawrie and V. V. Prudnikov, J. Phys. C 17 (1983) 1655; A. Weinrib and B. I. Halperin, Phys. Rev. B 27 (1983) 413; P.B. Weichman and K. Kim, Phys. Rev. B 40 (1989) 813.

[15] G. Grinstein, S. Ma and G. Mazenko, Phys. Rev. B 15 (1977) 258.

[16] E. R. Korutcheva and D. I. Uzunov, Phys. Lett 106A (1984) 175; G. Busiello, L. De Cesare, and I. Rabutto, Phys. Lett. 102A (1984) 41.

[17] J. A. Hertz, Phys. Rev. B 14 (1976) 1165; G. Busiello and L. De Cesare, Phys. Lett. 77A (1980) 177; D. I. Uzunov, Phys. Lett. 87A (1981) 11; A. Caramico D'Auria, L. De Cesare, and I. Rabutto, Physica A 225 (1996) 363. 


\section{Figure captions}

Fig.1. First-order diagram for $k_{0}^{\prime}$. The discontinuous line reprsents the disorder average $\left[|\varphi(\vec{k})|^{2}\right]_{R}=\Delta$ and the continuous line represents the bare correlation function $G_{0}\left(k_{0}, \vec{k}+\vec{k}_{1}\right)$.

Fig.2. Stability domains of $R$ for $n=4: R_{R}$ (triangle $2 a 4$ ) and $R_{C}(4 a b c)$.

Fig. 3. Stability domains of $H$ (triangle $2 h 4$ ) and $R$ for $n>4$. $R_{R}$ is given by the triangle $h 4 a$ and $R_{C}$ is the rectangle $a 4 c b$. The dotted line $b 4$ marks the extension of the stability domain of $H$ for $n=16$. The shaded region is explained in the text.

Fig. 4. Stability domains of $H$ and $R$ for $n=\infty$ (Hartree limit [10]). $R_{R}$ and $R_{C}$ are given by the triangles $h 4 a$ and $a 4 c$, respectively. 